\title{
Horizontal accuracy assessment of a novel algorithm for approximate a surface to a DEM
}

\author{
Barrera, D. ${ }^{\text {a,b }}$, Ibáñez, M.J. a , Eddargani, S. ${ }^{\text {a c }}$, Romero, R. ${ }^{\text {d }}$, Ariza-López, F.J. ${ }^{\text {e }}$ Reinoso-Gordo, \\ J.F. ${ }^{*}{ }^{*}$ \\ ${ }^{a}$ Department of Applied Mathematics, University of Granada, Spain-dbarrera@ugr.es, mibanez@ugr.es, \\ seddargani@correo.ugr.es \\ ${ }^{b}$ IMAG -- Institute of Mathematics, Granada, Spain \\ ${ }^{c}$ University Hassan First, MISI Laboratory, Morocco \\ ${ }^{d}$ Department of Computer Science and Artificial Intelligence, University of Granada, Spain-rocio@decsai.ugr.es \\ e Department of Cartographic, Geodesic and Photogrammetry Engineering-fjariza@ujaen.es \\ ${ }^{f}$ Department of Architectural and Engineering Graphic Expression - jreinoso@ugr.es \\ *jreinoso@ugr.es
}

\begin{abstract}
:
This study evaluates the horizontal positional accuracy of a new algorithm that defines a surface that approximates DEM data by means of a spline function. This algorithm allows evaluating the surface at any point in its definition domain and allows analytically estimating other parameters of interest, such as slopes, orientations, etc. To evaluate the accuracy achieved with the algorithm, we use a reference DEM $2 \mathrm{~m} \times 2 \mathrm{~m}\left(\mathrm{DEM}_{\mathrm{ref}}\right)$ from which the derived DEMs are obtained at $4 \mathrm{~m} \times 4 \mathrm{~m}, 8 \mathrm{~m} \times 8 \mathrm{~m}$ and $16 \mathrm{~m} \times 16 \mathrm{~m}\left(\mathrm{DEM}_{\mathrm{der}}\right)$. For each $\mathrm{DEM}_{\mathrm{der}}$ its spline approximant is calculated, which is evaluated at the same points occupied by the DEM $_{\text {ref }}$ cells, getting a resampled DEM $2 \times 2 m\left(D_{\text {EM }}\right)$. The horizontal accuracy is obtained by computing the area amongs the homologous contour lines derived from $\mathrm{DEM}_{\mathrm{ref}}$ and DEM $\mathrm{M}_{\text {rem, }}$ respectively. It has been observed that the planimetric errors of the proposed algorithm are very small, even in flat areas, where you could expect major differences. Therefore, this algorithm could be used when an evaluation of the horizontal positional accuracy of a DEM product at lower resolution $\left(\mathrm{DEM}_{\mathrm{pro}}\right)$ and a different producing source than the higher resolution $\mathrm{DEM}_{\mathrm{ref}}$ is wanted.
\end{abstract}

Keywords: Spline, Bernstein basis, control points, Bézier ordinates, tensor product, resample, horizontal accuracy, DEM

\section{Introduction}

Having a mathematical function that represents the terrain throughout its continuous definition domain has different advantages, among others the following: a) It is possible to sample regular meshes to generate digital elevation models (DEM) of both higher and lower resolution than the data from which the mathematical function was obtained; and this is achieved thanks to its definition domain is continuous, b) morphological variables of interest can be obtained from the corresponding mathematical formulas of the surfaces, such as slope, orientation, curvature and normal direction, c) You could intersect two surfaces corresponding to homologous DEMs from different dates and calculate the increase or decrease in the terrain volume. The provision of functions of this type has allowed resampling through bilinear (Maune, 2007) and bicubic (Keys, 1981) interpolations that have been used in different applications both to obtain DEMs of higher and lower resolution. In the first case, obtaining higher resolution has been used to, for example, improve urban flood zones in the absence of denser models (Shen and Tan, 2020); in the second case, its use has been frequent when it was intended to compare the altimetric accuracy of a lower resolution product model with another higher resolution reference model (Gao, 1998, Mukherjee et al. 2013, Wang et al. 2015), although, in most cases, the error introduced by the resampling from a higher resolution to a lower one was left unanalysed, as indicated by Mesa and Ariza, 2020. Other studies have addressed the influence of resampling techniques on products derived from DEMs such as streamflows. (Leong et al., 2015).

Although procedures are available to extract information directly from a DEM, we propose a new algorithm to build a surface with low computational cost that adjusts the elevations in order to have an explicit expression (function) from which to find the elements of interest. As the terrain has many irregularities, a surface should not be constructed too regular. $\mathrm{C} 1$ continuity is sufficient. The regular structure of the DEM allows to define a piecewise surface defined on a quadrangular partition of the terrain. More precisely, we define a piecewise bicubic surface by providing simple rules that give the Bézier ordinates (cf. Farin 2001) of the surface patches relative to the squares that make up the partition.

Most studies on DEM accuracy of DEM THT compare a product with a reference have analyzed the altitude 
component, leaving the horizontal component untouched. The reason for the lower number of investigations devoted to the horizontal component is due to the difficulty of finding a satisfactory method. In this work we will study the horizontal accuracy achieved by the new algorithm that we have proposed. We will use the automatic algorithm based on homologous contour lines introduced in Reinoso, 2010 and rigorously demonstrated in Reinoso, 2011 for evaluating the horizontal accuracy.

\section{Material and Methodology}

Our study was carried out on a $2 \times 2 \mathrm{~m}$ resolution $\mathrm{DEM}_{\text {ref }}$ produced by the Instituto Geográfico Nacional of Spain for the Navarra region. The following phases have been carried out:

1. Starting from the $2 \times 2 \mathrm{~m}$ resolution $\mathrm{DEM}_{\mathrm{ref}}$, a resampling has been performed using the nearest neighbor method at resolutions of 16x16,8x8 and $4 \times 4 \mathrm{~m}\left(\mathrm{DEM}_{\mathrm{der}}\right)$ which we respectively denote $\mathrm{DEM}_{4 \times 4}, \mathrm{DEM}_{8 \times 8}, \mathrm{DEM}_{16 \times 16}$. In this way, the $D_{\text {DEr }}$ have the same values as the DEM ${ }_{\text {ref }}$ at the points interpolated by the nearest neighbor method.

2. To evaluate the capacity of our approximation algorithm $\left(\mathrm{A}_{\mathrm{apx}}\right)$ to estimate the $\mathrm{DEM}_{\text {ref }}$ values, the surface of each $\mathrm{DEM}_{\text {ref }}$ se ha calculado la superficie de cada $\operatorname{DEM}_{\text {der }}\left(S_{\text {der }}\right)$ has been calculated using $A_{\text {apx }}$. $S_{\text {der }}$ is assessed to obtain DEMs with the same resolution as DEM $_{\text {ref }}$ which we call $\mathrm{DEM}_{\mathrm{remXxX}}\left(\mathrm{DEM}_{\mathrm{rem} 4 \times 4}, \mathrm{DEM}_{\mathrm{rem} 8 \times 8}\right.$, DEM $_{\text {rem16x16). }}$.

3. The horizontal displacement between $\mathrm{DEM}_{\mathrm{ref}}$ and each one of the $\mathrm{DEM}_{\mathrm{remXxX}}$ is calculated using the Reinoso 2011 contour method.

4. The results obtained with $\mathrm{A}_{\mathrm{apx}}$ are compared with the traditional bicubic resampling algorithm.

\subsection{Material}

The $\mathrm{DEM}_{\text {ref }}$ has a cell size of $2 \times 2 \mathrm{~m}$ and y occupies an area of $4.8 \times 4.8 \mathrm{Km}$. Figure 1 shows the geographical characteristics of the environment, as well as the DEM $\mathrm{Def}_{\mathrm{re}}$ that contains flat areas along with other steep slopes. The coordinates are referred in the ETRS89 system 30N UTM zone.
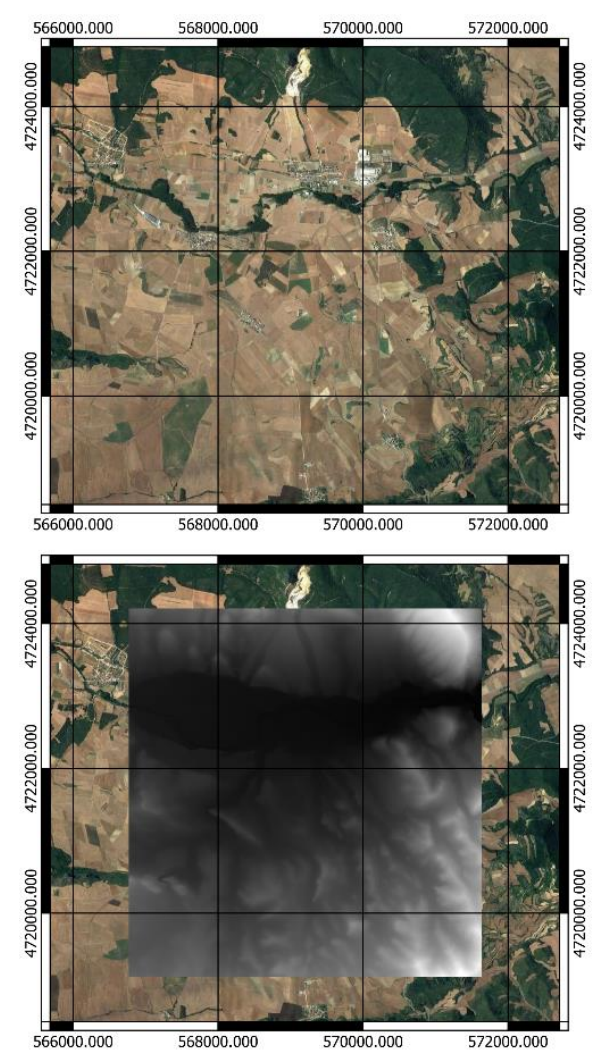

Figure 1: DEM used as reference and its geographical environment

\subsection{The approximation algorithm}

We propose to construct a spline surface by means of a tensor product of 1D spline approximants, defining the surface patches directly in the Bernstein basis. Suppose that for a real function $f$ the values $f\left(v_{i}\right), i \in \mathbb{Z}$, are known, where $v_{i}=i h$, with $h>0$ the size of the partition $\Delta:=$ $\left\{v_{i}: i \in \mathbb{Z}\right\}$. The $1 \mathrm{D}$ approximating spline $A f$ reduces on each interval $I_{i}:=\left[v_{i}, v_{i+1}\right]$ to a cubic polynomial, whose control polygon is formed by four control points with Bézier abscissae $\left\{v_{i}, v_{i}+\frac{h}{3}, v_{i+1}-\frac{h}{3}, v_{i+1}\right\}$. If $D_{3}$ is the union (without repetitions) of these Bézier abscissae and $u_{i}=v_{i}-\frac{h}{3}$ y $w_{i}=v_{i}+\frac{h}{3}$, then $D_{3}=\bigcup_{i \in \mathbb{Z}}\left\{u_{i}, v_{i}, w_{i}\right\}$.

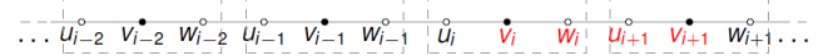

For $x \in I_{i}$, it holds

$A f(x)=V_{i} B_{0}(t)+W_{i} B_{1}(t)+U_{i+1} B_{2}(t)+V_{i+1} B_{3}(t)$, with $t=\frac{x}{h}-i$ and $B_{k}(t)=\left(\begin{array}{l}3 \\ k\end{array}\right) t^{k}(1-t)^{3-k}, 0 \leq k \leq 3$. The Bézier ordinates $V_{i}, W_{i}, U_{i+1}$ and $V_{i+1}$ are defined as linear combinations of point values $f\left(v_{i}\right): V_{i}=f\left(v_{i}\right)$,

$$
U_{i}=\sum_{r=-1}^{1} \alpha_{r} f\left(v_{i+r}\right), \quad W_{i}=\sum_{r=-1}^{1} \beta_{r} f\left(v_{i+r}\right),
$$

where the masks $\alpha=\left(\alpha_{-1}, \alpha_{0}, \alpha_{1}\right) \in \mathbb{R}^{3}$ and $\beta=$ $\left(\beta_{-1}, \beta_{0}, \beta_{1}\right) \in \mathbb{R}^{3}$ are determined to achieve $C^{1}$ continuity as well as the reproduction of the quadratic polynomials. 
Proposition 1 The unique masks yielding $C^{1}$ continuity and the reproduction of quadratic polynomials are

$$
\alpha=\left(\frac{1}{6}, 1,-\frac{1}{6}\right) \text { and } \beta=\left(-\frac{1}{6}, 1, \frac{1}{6}\right) \text {. }
$$

Furthermore, the uniform norm of the corresponding operator $A$ is equal to $4 / 3$.

From the exactness of $A$, the following result regarding the approximation error holds.

Proposition 2 There exist constans $K_{l}, l=0,1$, independent of $f$ and $h$ and $i$, such that

$$
\left\|(f-A f)^{(l)}\right\|_{\infty, I_{i}} \leq K_{l} h^{3-l}\left\|f^{(3)}\right\|_{\infty, \Omega^{\prime}},
$$

where $\Omega=\left[v_{i-1}, v_{i+2}\right]$.

Now, given a 2D function $f(x, y)$ a bi-cubic piecewise surface $s$ is defined as a tensor product approximant: the operator $A$ is applied to $f$ as a function depending on $x$ (or $y$ ), and then $A$ is again applied to de resulting function, i.e. $s(x, y)=A_{y} A_{x} f(x, y)$.

On each square $I_{i} \times I_{j}$ this function is a bi-cubic Bézier surface, so that it can be represented in Bernstein-Bézier form. It is a linear combination of functions

$$
B_{m}\left(\frac{x}{h}-i\right) B_{n}\left(\frac{x}{h}-j\right), 0 \leq m, n \leq 3,
$$

whose coefficients are expressed in terms of values $f\left(v_{i+k}, v_{j+l}\right),-1 \leq k, l \leq 1$.

For Franke function (see Franke 1982) (top), the results provided by the method proposed for $h=2^{-7}$ are shown in Fig. 2.
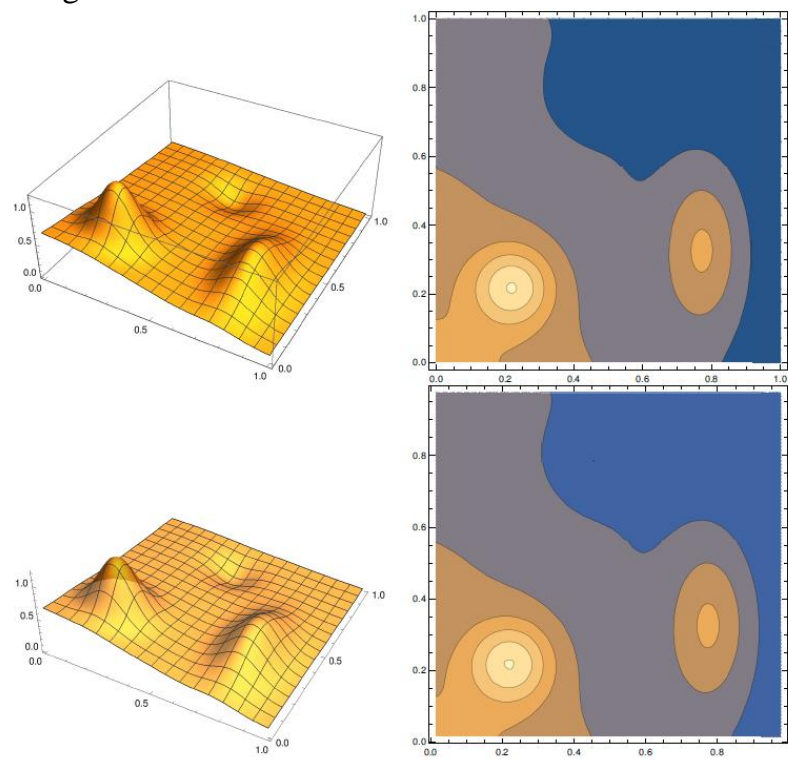

Figure 2: Results provided by our $A_{\text {apx }}$ for $h=^{2-7}$ according to the Franke funcion
Also for Nielson test function (Nielson 1978) it provides good results for the same step length, shown in Fig.3.
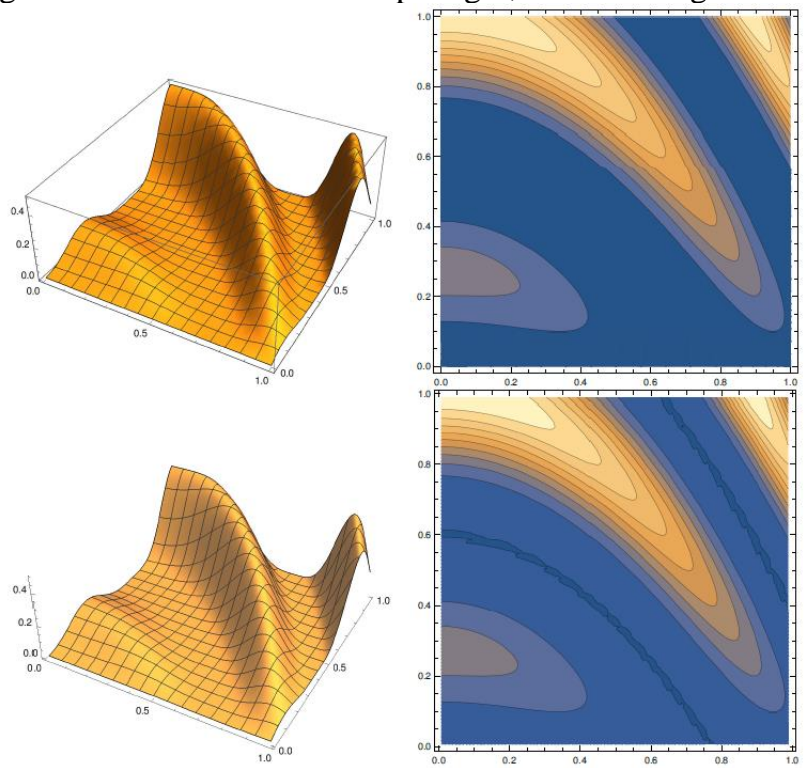

Figure 3: Results from our $A_{\text {apx }}$ algorithm according to the Nielson test function

\subsection{Contours-based algorithm to measure the horizontal displacement}

The the horizontal displacement computation of the fases with respect to the $\mathrm{DEM}_{\text {ref }}$ is carried out in the following phases:

1. The contours of both DEMs are calculated (Fig. 4 $\mathrm{a}$ and $\mathrm{b}$ respectively), and their homologous curves are automatically identified, e.g. curves $\mathrm{C}_{\mathrm{a}}$ and $\mathrm{C} 4_{\mathrm{b}}$ in Fig. $4 \mathrm{a}$ and $\mathrm{b}$.

2. After superimposing the homologous contours (Fig. 4 c), the areas enclosed between them are calculated (gray area in Fig. 4 d). The horizontal displacement (Hdi) computed by the ith pair of homologous contours $\left(\mathrm{Ci}_{\mathrm{a}}, \mathrm{Ci}_{\mathrm{b}}\right)$ is formulated as the area enclosed by both curves (Ai) divided by the mean length of those contours $\left(L m_{i}=\right.$ $\left.\frac{L i_{a}+L i_{b}}{2}\right)$

$$
H d_{i}=\frac{A_{i}}{L m_{i}}
$$

3. And the mean displacement of the $D_{\text {EM }} M_{\text {remXxX }}$ respect to $\mathrm{DEM}_{\text {ref }}\left(H d_{\mathrm{DEMremXxX}}\right)$ is computed as the weighted average of the displacements of all the homologous contours, the weighting factor being the average length of those contours, divided by the total length of the average contours being the total length $L_{T o t}=\sum_{i=1}^{n} L m_{i}$ :

$$
H d_{\text {DEMremXxX }}=\frac{1}{L_{\text {Tot }}} \sum_{i=1}^{n} A_{i} * L m_{i}
$$



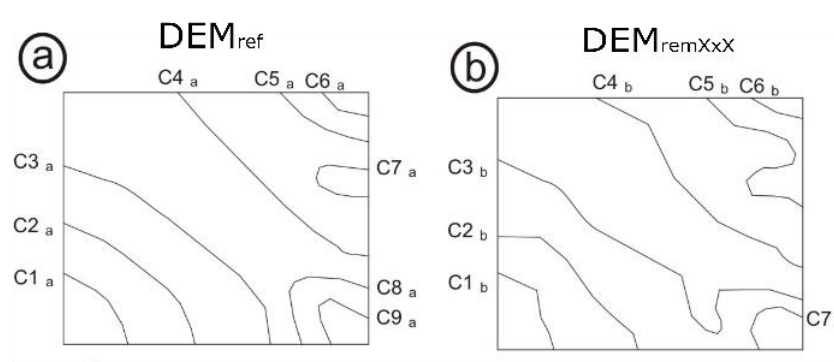

(C)

DEM $_{\text {ref }} \&$ DEM $M_{r e m \times x x}$
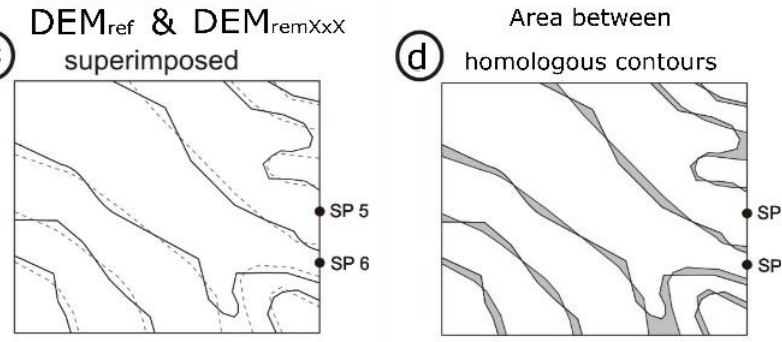

Figure 4: Hologous contours and area between them

\section{Results and discussion}

To calculate the $H d_{\text {DEMremXxx }}$ a $10 \mathrm{~m}$ interval between contour lines has been used, that in our DEM produces a total of 22 levels, specifically their heights ranging from 450 to $660 \mathrm{~m}$. In Fig. 5 you can see the homologous contour drawn on a shadow map, as well as a detail where the area enclosed between those homologous contours are highlighted on green color.

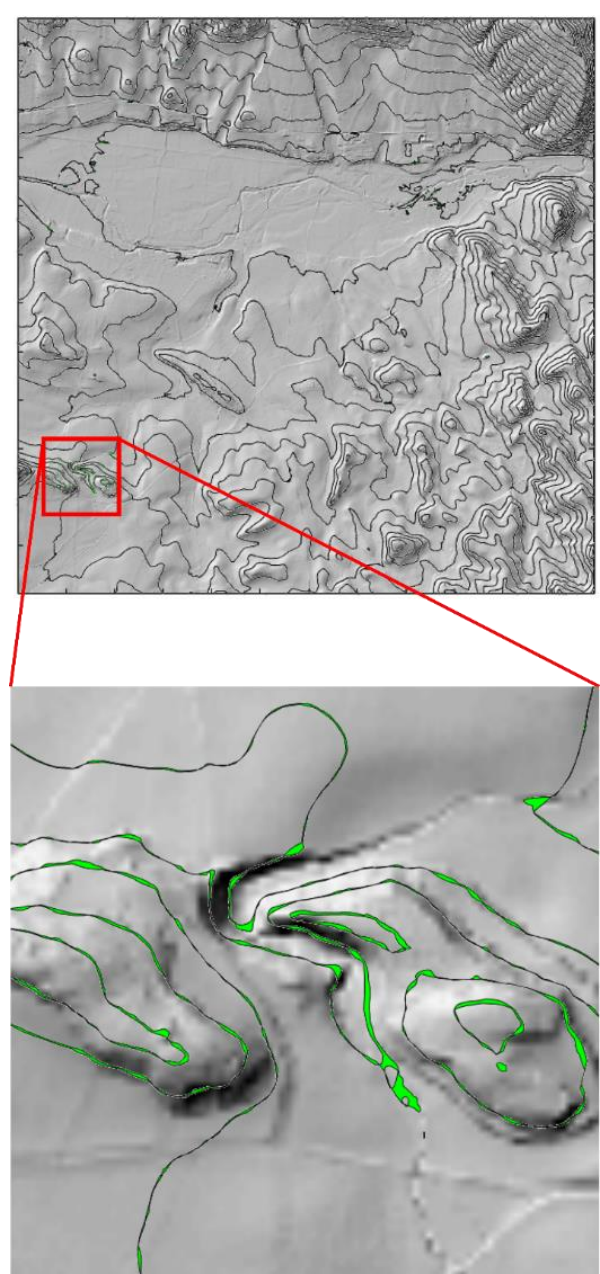

Figure 5: Area between homologous contours corresponding to the DEMref and the DEMreml6x16

Table 1 shows the mean horizontal displacements as well as their standard deviations calculated for $4 \times 4,8 \times 8$ and $16 \times 16 \mathrm{~m}$ resolutions using our new $\mathrm{A}_{\mathrm{apx}}$ algorithm and the traditional bicubic resampling method.

\begin{tabular}{|c|c|c|c|}
\hline $\mathrm{DEM}_{\mathrm{remXxX}}$ & $H d_{\text {DEMremXxX }}$ & $\mathrm{A}_{\mathrm{apx}}(\mathrm{m})$ & $\begin{array}{l}\text { Bicubic } \\
\text { (m) }\end{array}$ \\
\hline \multirow{2}{*}{$\mathrm{DEM}_{\mathrm{rem} 4 \times 4}$} & Mean & 0.07 & 0.99 \\
\hline & Std & 0.03 & 0.06 \\
\hline \multirow{2}{*}{$\mathrm{DEM}_{\text {rem } 8 \times 8}$} & Mean & 0.28 & 1.05 \\
\hline & Std & 0.13 & 0.06 \\
\hline \multirow{2}{*}{$\mathrm{DEM}_{\mathrm{rem} 16 \times 16}$} & Mean & 0.81 & 1.38 \\
\hline & Std & 0.38 & 0.27 \\
\hline
\end{tabular}

Table 1 shows that our new algorithm produces better results than the traditional bicubic algorithm regardless of

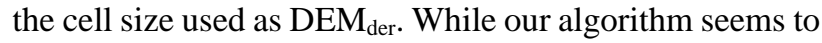
decrease the error due to horizontal displacement at a rate of $1 / 4$ as the resolution increases at a rate of 2 , in the bicubic algorithm the rate of decrease is much lower. However, no large differences are observed in the values of the standard deviations if both algorithms are compared for each level 
of resolution. However, additional tests should be carried out with a greater number of DEMs, covering all types of terrain (flat, undulating and mountainous), in order to statistically validate the apparently better results or our algorithm respect the traditional bicubic one.

Another advantage of the new algorithm with respect to the traditional bicubic one is that it has a lower computational cost, making it a candidate to be implemented in cartographic production software packages.

We believe that this new algorithm can be used when you want to know the positional accuracy (horizontal and vertical) of a lower resolution DEM coming from a source other than the reference one or that has been created with a different method from the reference one.

On the other hand, it would also be interesting to have an algorithm that not only reported the horizontal displacement with a scalar value, but also included information about direction in each of the cells, which would be possible by adapting the contours method by Reinoso 2011.

\section{Conclusions}

In this work a new algorithm $\left(\mathrm{A}_{\mathrm{apx}}\right)$ is presented to approximate a DEM by means of a piecewise defined surface $\left(S_{\text {der }}\right)$. It presents some advantages linked to its definition type, such as being able to obtain the altitude of a point in the entire definition domain of that surface, as well as morphological variables that characterize the terrain surface: slope, orientation, curvature or normal direction in an analytical way. An immediate application would be the possibility of resampling $S_{\text {der }}$ to obtain DEMs $\left(\mathrm{DEM}_{\mathrm{remXxx}}\right)$ of higher or lower resolution than those used to create $S_{\text {der }}$. One consequence of $A_{\text {apx }}$ resampling capabilities is being able to assess the accuracy of a product DEM (DEMpro) against a higher accuracy

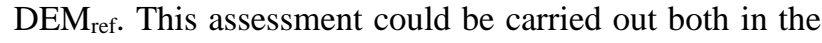
vertical component and in the horizontal component, which is the one studied in this work. $\mathrm{DEM}_{\text {pro }}$ can come from both a source or a method other than the source or method used to create the DEM $\mathrm{Dref}_{\text {. }}$

$\mathrm{A}_{\text {apx }}$ has shown a lower horizontal displacement than the traditional bicubic interpolation algorithm, which can be interpreted as a lower error when resampling DEMs of lower resolution to others of higher resolution; These processes are necessary when trying to compare the accuracies of a $\mathrm{DEM}_{\text {pro }}$ against a $\mathrm{DEM}_{\text {ref }}$, and whenever possible it will be necessary to choose those algorithms that produce the least error (horizontal displacement).

The $\mathrm{A}_{\text {apx }}$ computational cost is lower than other conceptually similar such as the traditional bicubic one.

Finally, in the future an experiment will have to be designed with a sufficiently large number of DEMs on which to test $A_{\text {apx }}$ so that the results that appear in this first $\mathrm{A}_{\text {apx }}$ study can be verified.

\section{References}

Farin, G. (2001). Curves and Surfaces for CAGD: A practical guide, 5th Edition. Morgan Kaufmann, San Francisco, USA.

Franke, R. (1982) Scattered data interpolation: tests of some methods, Math. Comp. 157, pp. 181-200.

Gao, J. (1998). Impact of sampling intervals on the reliability of topographic variables mapped from grid DEMs at a micro-scale. Int. J. Geogr. Inf. Sci., 12, 875890.

Keys, R. (1981). "Cubic convolution interpolation for digital image processing". IEEE Transactions on Acoustics, Speech, and Signal Processing. 29 (6): 11531160

Leong Tan, M.; Ficklin, D.L.; Dixon, B.; Ibrahim, A.L.; Yusop, Z.; Chaplot, V. (2015). Impacts of DEM resolution, source, and resampling technique on SWATsimulated streamflow. Appl. Geogr., 63, 357-368

Maune D (2007) Digital elevation model technologies and applications: the DEM user's manual, 2nd edn.Bethesda, USA

Mesa-Mingorance, J.L.; Ariza-López, F.J. (2020) Accuracy Assessment of Digital Elevation Models (DEMs): A Critical Review of Practices of the Past Three Decades. Remote Sens. , 12, 2630.

Mukherjee, S.; Joshi, P.K.; Mukherjee, S.; Ghosh, A.; Garg, R.D.; Mukhopadhyay, A (2013). IU accuracy of open source Digital Elevation Model (DEM). Int. J. Appl. Earth Obs. Geoinf., 21, 205-217.

Nielson G. M. (1978) A first order blending method for triangles based upon cubic interpolation, Int. J. Numer. Meth. Engrg. 15, pp. 308-318.

Reinoso, J. F. (2010) A priori horizontal displacement (HD) estimation of hydrological features when versioned DEMs are used, Journal of Hydrology,384: 1-2, pp. 130141.

Reinoso, J. F. (2011). An algorithm for automatically computing the horizontal shift between homologous contours from DTMs, ISPRS Journal of Photogrammetry and Remote Sensing, 66: 3, pp. 272-286.

Shen, J., Tan, F. (2020). Effects of DEM resolution and resampling technique on building treatment for urban inundation modeling: a case study for the 2016 flooding of the HUST campus in Wuhan. Nat Hazards 104, 927957.

Wang, B.; Shi, W.; Liu, E. (2015). Robust methods for assessing the accuracy of linear interpolated DEM. Int. J. Appl. Earth Obs. Geoinf., 34, 198-206. 